\title{
CONTATO PELE-A-PELE MÃE/FILHO NA PRIMEIRA HORA DE VIDA: UMA REVISÃO INTEGRATIVA
}

\section{SKIN-TO-SKIN CONTACT BETWEEN MOTHER AND CHILD IN THE FIRST HOUR OF LIFE: AN INTEGRATIVE REVIEW}

\author{
Poliana Lopes Campos de Sá ${ }^{1}$ Erika Marina Rabelo ${ }^{2}$
}

\section{RESUMO}

Objetivo: identificar nas evidências disponíveis quais fatores maternos, profissionais e pessoais estão relacionados à realização do contato pele a pele mãe/filho na primeira hora de vida do recémnascido e analisá-los de acordo com o que é recomendado pelo Ministério da Saúde e Organização Mundial de Saúde. Método: revisão integrativa com 19 artigos publicados entre os anos de 2014 e 2019 nas bases de dados MEDLINE, LILACS e SciELO, utilizando trabalhos disponíveis na íntegra. Resultados: foram criadas duas categorias de análise: fatores comportamentais e fatores fisiológicos. Conclusão: Os fatores associados ao contato pele-a-pele encontrados a partir dessa revisão foram: vínculo materno infantil, início e duração da amamentação, preparo da equipe atuante em sala de parto, parto vaginal, bem-estar materno e infantil no pós-parto imediato, termorregulação neonatal, redução de estresse e dor do binômio e prevenção de hemorragia no pósparto.

Palavras-chave: Contato Pele-a-Pele. Aleitamento Materno. Relações Mãe-Filho. Período PósParto.

\begin{abstract}
Objective: to identify in the available evidence which maternal, professional and personal factors are related to the skin-to-skin contact between mother and child in the first hour of life of the newborn and to analyze them according to what is recommended by the Ministry of Health and World Health Organization. Method: integrative review with 19 articles published between 2014 and 2019 in the MEDLINE, LILACS and SciELO databases, using works available in full. Results: two categories of analysis were created: behavioral factors and physiological factors. Conclusion: The factors associated with skin-to-skin contact found from this review were: maternal infant bond, beginning and duration of breastfeeding, preparation of the team working in the delivery room, vaginal delivery, maternal and child well-being in the postpartum period. immediate delivery, neonatal thermoregulation, reduction of binomial stress and pain and prevention of postpartum hemorrhage.
\end{abstract}

Keywords: Skin-to-Skin Contact. Breastfeeding. Mother-Child Relations. Postpartum Period.

\footnotetext{
${ }^{1}$ Fundação Hospitalar do Estado de Minas Gerais. Orcid: https://orcid.org/0000-0001-8127-1977. E-mail: polianasa.enf@ gmail.com ${ }^{2}$ Fundação Hospitalar do Estado de Minas Gerais. Orcid: http://orcid.org/0000-0002-8616-1977. E-mail: erikamarinarabelo@yahoo.com.br
} 


\section{INTRODUÇÃO}

Os cuidados prestados ao bebê assim que ele nasce são essenciais para a sua adaptação diminuindo a morbimortalidade neonatal. O momento de transição do meio intra para o extrauterino é bem delicado porque é marcado por inúmeras mudanças para a criança. $\mathrm{O}$ meio intrauterino oferece aconchego, temperatura, ruídos e luminosidade confortáveis e lá não é necessário esforço para realizar as funções vitais. Quando nasce, o bebê vai superando, aos poucos, as dificuldades pertencentes ao seu desenvolvimento. A sua adaptação ao meio extrauterino é de forma gradativa ${ }^{(1)}$.

É recomendação da Organização Mundial de Saúde (OMS) que todos os bebês recebam cuidados neonatais essenciais durante o parto e nascimento visto que são cuidados que reduzem consideravelmente os índices de mortes neonatais evitáveis. Um dos cuidados essenciais ao neonato é a proteção térmica através de uma prática denominada contato pele-a-pele $(\mathrm{CPP})^{(2)}$.

Contato pele-a-pele entre mãe e recémnascido é quando o bebê, imediatamente após o parto, é colocado sem roupa sobre o peito desnudo da mãe, e assim os dois permanecem por pelo menos 60 minutos cobertos com um campo aquecido. É uma técnica barata e facilmente executada mesmo em hospitais pequenos e em países subdesenvolvidos, e além de tudo promove inúmeros benefícios à díade mãe-filho ${ }^{(3)}$.
Nas situações em que não são necessários cuidados imediatos de urgência ao recémnascido ou à mulher, recomenda-se que logo após o nascimento as mulheres sejam estimuladas a terem CPP imediato, sendo a criança coberta com um campo ou toalha para que não perca calor enquanto mantém o contato. Deve-se evitar o afastamento de mãe e filho na primeira hora após o nascimento para realização de procedimentos de rotina tais como medir, pesar e dar banho ${ }^{(4)}$.

É válido perguntar à mãe se ela deseja ou não ter seu filho junto a ela assim que parir. A mulher tem o direito de escolher sobre isso. Para tanto, é necessário que antes do parto a equipe de saúde responsável por essa mulher a explique as vantagens do CPP e tudo que este ato envolve. Dessa forma, a mulher estará participando ativamente do momento e poderá decidir de forma mais consciente ${ }^{(5)}$.

O CPP e a amamentação na primeira hora de vida têm sido propagados como práticas de humanização do atendimento à mulher no seu parto, e o seu objetivo é promover a qualidade da assistência prestada na sala de parto e o aleitamento materno ${ }^{(6)}$.

Existe uma estratégia da OMS e do Fundo das Nações Unidas para a Infância (UNICEF) lançada no ano de 1991 que objetiva capacitar os funcionários de hospitais e maternidades para apoio, promoção e proteção ao aleitamento materno. Esta estratégia recebe o nome de Iniciativa Hospital Amigo da Criança $(\text { IHAC })^{(7)}$. OMS e UNICEF 
recomendam que a amamentação seja exclusiva durante os primeiros seis meses de vida, começando dentro da primeira hora após o nascimento ${ }^{(8)}$.

Um dos critérios da IHAC compõe-se da adesão aos "Dez Passos para o Sucesso do Aleitamento Materno" pelas maternidades certificadas e, no caso do Brasil, à Norma Brasileira de Comercialização de Alimentos para Lactentes e Crianças de Primeira Infância, Bicos, Chupetas e Mamadeiras (NBCAL). Os Dez Passos são recomendações que beneficiam a amamentação através de orientações desde o período pré-natal até o retorno ao $\operatorname{lar}^{(7)}$.

O $4^{\circ}$ destes Dez Passos para o Sucesso do Aleitamento Materno orienta "colocar os bebês em contato pele a pele com suas mães imediatamente após o parto durante pelo menos uma hora e encorajar as mães a reconhecerem quando seus bebês estão prontos para mamar, oferecendo ajuda, se necessário". As únicas justificativas para retardar esse contato são questões de saúde materna e/ou neonatal. Se tais questões não existirem, todas as mães devem ter seus filhos colocados em CPP imediatamente permanecendo juntos por pelo menos uma $\operatorname{hora}^{(9)}$.

O CPP imediatamente após o nascimento até que acabe a primeira amamentação traz inúmeros benefícios. Foi demonstrado que aumenta as chances de se prolongar a duração da amamentação e também melhorar as taxas de aleitamento materno exclusivo ${ }^{(8)}$.

A amamentação e o CPP associados na primeira hora de vida estimulam a produção de leite materno, incluindo colostro, também chamado de "primeira vacina" do bebê que é rica em nutrientes e anticorpos ${ }^{(10)}$.

Deve-se estimular a amamentação na primeira hora de vida, mas isso não é sinônimo de forçar os bebês contra a mama. A mãe e o bebê devem ser mantidos de forma tranquila e serena em CPP até que ambos se sintam prontos para o aleitamento, e o tempo para que isso aconteça é indeterminado. $\mathrm{O}$ toque no mamilo e na aréola logo após o parto faz com que o organismo libere ocitocina que por sua vez é um hormônio que ajuda com que a contração uterina ocorra mais rapidamente, o que pode controlar o sangramento e prevenir hemorragia pós-parto. A liberação de ocitocina também faz a mãe sentir mais afeto pelo bebê, tornando-a assim, mais ligada a ele $e^{(9,11)}$.

A espécie humana nasce programada para criar com a mãe uma conexão que ultrapassa as dimensões fisiológicas. O primeiro contato entre mãe e recém-nascido é o momento que as competências afeto-emocionais, que serão a base para o desenvolvimento do apego e do vínculo, começam a surgir. Por isso e para isso, nos casos em que a amamentação é contraindicada o CPP deve ser encorajado da mesma forma ${ }^{(3,12)}$. 
Muitas pesquisas têm comprovado os benefícios fisiológicos e psicossociais do CPP para a saúde da mãe e do seu recém-nascido. Segundo a neurociência da espécie mamífera, este contato gera comportamentos neurais que garantem plena satisfação de necessidades biológicas básicas. Esse primeiro instante depois do parto pode ser um período importante para o comportamento futuro do organismo do bebê $\hat{e}^{(3,13)}$.

O estudo justifica-se pela execução de um projeto guarda-chuva da linha de pesquisa Saúde Materna e Perinatal da Maternidade Odete Valadares (MOV) em Belo Horizonte MG, desde o ano de 2019, que objetiva avaliar a prática do CPP na visão de puérperas, gestores e trabalhadores e observação da técnica do CPP. As informações geradas na presente revisão integrativa irão subsidiar a execução das outras etapas do estudo guarda-chuva com evidências científicas recentes nacionais e internacionais, fomentando assim, novas discussões e propostas de melhorias na prática do CPP.

Para atingir o objetivo proposto, procurou-se responder a seguinte questão: Quais fatores estão associados à realização do CPP mãe/filho na primeira hora de vida?

Logo, objetivou-se identificar nas evidências disponíveis quais fatores maternos, profissionais e pessoais estão relacionados à realização do CPP mãe/filho na primeira hora de vida do recém-nascido.

\section{MÉTODO}

IN DERME

Trata-se de uma revisão integrativa acerca do que há publicado sobre o CPP mãe/bebê na primeira hora de vida e o que está associado a esta prática. Este tipo de estudo proporciona síntese de conhecimento e permite que resultados de estudos significativos sejam aplicados na prática, além de se tratar de uma metodologia que é firmada na Prática Baseada em Evidências $(\mathrm{PBE})^{(14)}$.

A PBE caracteriza-se como abordagem que envolve a elucidação de um problema, a procura e avaliação crítica das evidências encontradas, implementação das evidências na prática e avaliação dos resultados que forem obtidos ${ }^{(15)}$.

Este estudo foi construído seguindo as seis etapas da revisão integrativa: elaboração da pergunta norteadora da pesquisa; busca na literatura; coleta de dados; análise crítica dos estudos que foram incluídos; discussão dos resultados; e por último, apresentação final do $\operatorname{artigo~de~revisão~integrativa~}^{(14)}$.

O levantamento de artigos foi feito nas bases de dados Medical Literature Analysis and Retrieval System Online (MEDLINE), Literatura Latino-Americana e do Caribe em Ciências da Saúde (LILACS) e na Scientific Electronic Library Online (SciELO). $\mathrm{Na}$ estratégia de busca criteriosa dos artigos, utilizou-se a palavra-chave contato pele-apele e os descritores extraídos dos Descritores em Ciências da Saúde (DeCS) e do Medical Subject Headings (MeSH): aleitamento https://doi.org/10.31011/reaid-2021-v.95-n.35-art.1079 Rev Enferm Atual In Derme v. 95, n. 35, 2021 e-021120 
materno, relações mãe-filho e período pósparto bem como as suas versões em inglês e espanhol. Justifica-se o uso de uma palavrachave visto que o termo 'contato pele-a-pele' ainda não é apresentado como um descritor em saúde e foi objeto deste estudo. A fim de deixar a busca mais ampla, os termos foram combinados de diferentes maneiras e fez-se o uso do operador booleano AND para ocorrência de assuntos de forma simultânea.

Foram incluídos artigos científicos disponíveis na íntegra e publicados nos idiomas português, inglês ou espanhol no período de 2014 a 2019. Este recorte temporal justifica-se pelo interesse dos autores em analisarem estudos recentes publicados nos últimos cinco anos. A busca dos artigos ocorreu por meio de acesso on-line às bases de dados, nos meses de setembro de 2019 a fevereiro de 2020. Artigos que não estavam disponíveis em sua forma completa, que foram publicados fora do espaço temporal delimitado, os que eram repetidos em uma ou mais base de dados, e os que não condiziam com a temática foram considerados critérios de exclusão.
Para a seleção dos estudos, inicialmente foi feita a leitura de títulos e resumos. Em seguida, fez-se a revisão por pares das publicações na íntegra, e assim foram selecionadas aquelas que continham os critérios de elegibilidade. A apuração dos dados dos estudos inclusos na revisão foi realizada por três revisores, de forma independente. Posteriormente, houve um debate entre os revisores e a orientadora da pesquisa e assim foram definidos os artigos que fariam parte da revisão integrativa.

$\mathrm{Na}$ busca inicial, com aplicação dos critérios de inclusão e exclusão e após leitura de título e resumo, foi possível encontrar um total de 29 estudos publicados. Após leitura na íntegra, 10 estudos foram excluídos, pois não atendiam ao problema da pesquisa. Dessa forma, 19 artigos científicos foram incluídos nesta revisão integrativa. A seleção foi de acordo com o protocolo Preferred Reportingn Items for Systematics Reviews and MetaAnalyses (PRISMA), conforme apresentado no Fluxograma 1. 
Fluxograma 1 - Processo de seleção dos artigos
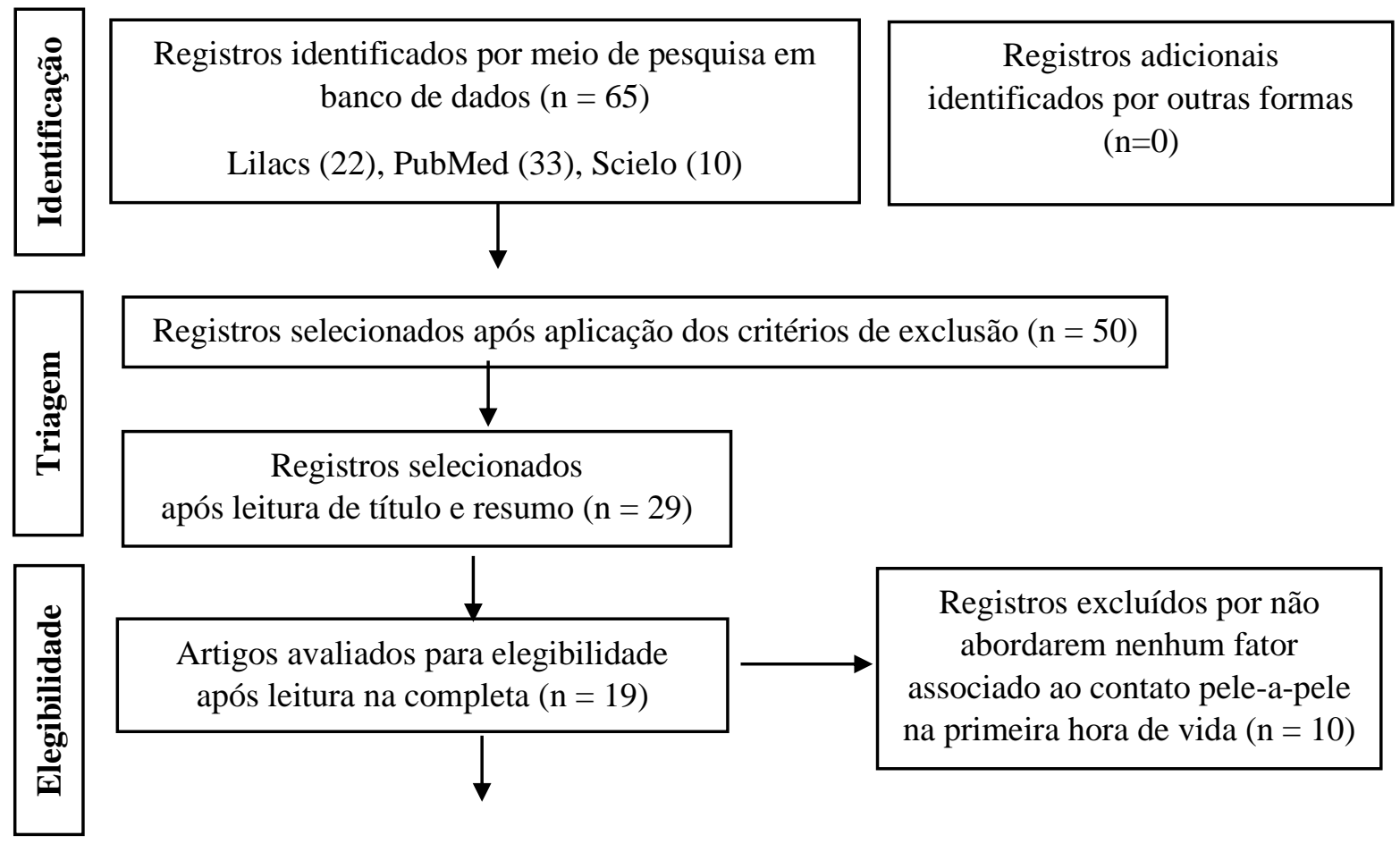

Registros excluídos por não abordarem nenhum fator associado ao contato pele-a-pele na primeira hora de vida $(\mathrm{n}=10)$
Estudos incluídos na revisão integrativa $(n=19)$

Fonte: Elaboração própria

Por fim, os artigos foram categorizados através dos níveis de evidência da seguinte forma: Nível 1: evidências decorrentes da meta-análise de múltiplos estudos clínicos controlados e randomizados; Nível 2: evidências arranjadas em estudos individuais com delineamento experimental; Nível 3: evidências de estudos quase-experimentais; Nível 4: evidências de estudos descritivos (não-experimentais) ou com abordagem qualitativa; Nível 5: evidências provindas de relatos de caso ou de experiência; Nível 6: evidências baseadas em opiniões de especialistas $^{(14)}$.

\section{Resultados}

Dezenove artigos constituíram a amostra final. O Quadro 01 apresenta um apanhado das principais informações extraídas dos estudos com base no objetivo de interesse desta revisão. 
Quadro 01 - Apresentação dos artigos segundo autores/ano de publicação em ordem crescente, título, tipo de estudo/nível de evidência e principais fatores associados ao CPP.

\begin{tabular}{|c|c|c|c|}
\hline $\begin{array}{c}\text { Autores/ } \\
\text { Ano }\end{array}$ & Título & $\begin{array}{c}\text { Tipo de estudo/ } \\
\text { Nível de evidência }\end{array}$ & $\begin{array}{l}\text { Fator associado ao } \\
\text { contato pele a pele }\end{array}$ \\
\hline $\begin{array}{l}\text { Santos LM. et al., } \\
2014\end{array}$ & $\begin{array}{l}\text { Vivenciando o contato pele a } \\
\text { pele com o recém-nascido no } \\
\text { pós-parto como um ato } \\
\text { mecânico }^{(16)} \text {. }\end{array}$ & $\begin{array}{l}\text { Estudo exploratório, } \\
\text { descritivo e } \\
\text { qualitativo. } \\
4\end{array}$ & $\begin{array}{l}\text { Atuação da equipe de } \\
\text { sala de parto. }\end{array}$ \\
\hline $\begin{array}{l}\text { Crenshaw JT; } \\
2014\end{array}$ & $\begin{array}{l}\text { Prática saudável do } \\
\text { nascimento \# 6: mantenha } \\
\text { mãe e bebê juntos - é melhor } \\
\text { para mãe, bebê e aleitamento } \\
\text { materno }^{(17)} \text {. }\end{array}$ & $\begin{array}{l}\text { Estudos descritivo } \\
\text { não-experimental } \\
4\end{array}$ & $\begin{array}{l}\text { Vínculo materno- } \\
\text { infantil; } \\
\text { Termorregulação } \\
\text { neonatal; } \\
\text { Início da amamentação; } \\
\text { Redução do estresse } \\
\text { materno. }\end{array}$ \\
\hline $\begin{array}{l}\text { Fucks IS. et al., } \\
2015\end{array}$ & $\begin{array}{l}\text { A sala de parto: o contato } \\
\text { pele a pele e as ações para o } \\
\text { estímulo ao vínculo entre } \\
\text { mãe-bebê } \\
(18) \text {. }\end{array}$ & $\begin{array}{l}\text { Estudo qualitativo de } \\
\text { caráter descritivo } \\
4\end{array}$ & $\begin{array}{l}\text { Vínculo materno- } \\
\text { infantil. }\end{array}$ \\
\hline $\begin{array}{l}\text { Vieira MJO; Dos } \\
\text { Santos AAP; } \\
\text { Silva JMO; } \\
\text { Sanches METO; } \\
2016\end{array}$ & $\begin{array}{l}\text { Assistência de enfermagem } \\
\text { obstétrica baseada em boas } \\
\text { práticas: do acolhimento ao } \\
\text { parto }^{(19)} \text {. }\end{array}$ & $\begin{array}{l}\text { Estudo descritivo, } \\
\text { retrospectivo e } \\
\text { documental, de } \\
\text { abordagem } \\
\text { quantitativa } \\
4\end{array}$ & $\begin{array}{l}\text { Vínculo materno- } \\
\text { infantil. }\end{array}$ \\
\hline $\begin{array}{l}\text { Albuquerque RS. } \\
\text { et al., } 2016\end{array}$ & $\begin{array}{l}\text { Temperatura dos recém- } \\
\text { nascidos submetidos ao calor } \\
\text { radiante e ao dispositivo Top } \\
\text { Maternal ao nascimento }\end{array}$ & $\begin{array}{l}\text { Estudo comparativo } \\
\text { observacional do } \\
\text { tipo Caso-Controle } \\
4\end{array}$ & $\begin{array}{l}\text { Termorregulação } \\
\text { neonatal. }\end{array}$ \\
\hline Sharma A; 2016 & $\begin{array}{l}\text { Eficácia do contato pele a } \\
\text { pele precoce na taxa de } \\
\text { aleitamento materno } \\
\text { exclusivo em recém-nascidos } \\
\text { a termo: um estudo } \\
\text { controlado randomizado } \\
\end{array}$ & $\begin{array}{l}\text { Estudo controlado } \\
\text { randomizado } \\
2\end{array}$ & $\begin{array}{l}\text { Aleitamento materno } \\
\text { exclusivo; } \\
\text { Redução de dor materna } \\
\text { durante suturas. }\end{array}$ \\
\hline $\begin{array}{l}\text { Silva CM; Pereira } \\
\text { SCL; Passos IR; } \\
\text { Dos Santos LC; } \\
2016\end{array}$ & $\begin{array}{l}\text { Fatores associados ao contato } \\
\text { pele a pele entre mãe/filho e } \\
\text { amamentação na sala de } \\
\text { parto }^{(22)} \text {. }\end{array}$ & $\begin{array}{l}\text { Estudo retrospectivo } \\
4\end{array}$ & $\begin{array}{l}\text { Aleitamento materno; } \\
\text { Parto vaginal. }\end{array}$ \\
\hline $\begin{array}{l}\text { Guala A. et al., } \\
2017\end{array}$ & $\begin{array}{l}\text { Contato pele a pele no parto } \\
\text { cesáreo e duração do } \\
\text { aleitamento materno: um } \\
\text { estudo de coorte }{ }^{(23)} \text {. }\end{array}$ & $\begin{array}{l}\text { Estudo de coorte } \\
3\end{array}$ & Cesariana. \\
\hline $\begin{array}{l}\text { Souza LH; Soler } \\
\text { ZASG; Santos } \\
\text { MLSG; Sasaki } \\
\text { NSGMS; } 2017\end{array}$ & $\begin{array}{l}\text { Puérperas se relacionando } \\
\text { com seus filhos e } \\
\text { experiências de trabalho }{ }^{(24)} \text {. }\end{array}$ & $\begin{array}{l}\text { Estudo transversal } \\
4\end{array}$ & $\begin{array}{l}\text { Vínculo materno- } \\
\text { infantil. }\end{array}$ \\
\hline Linares AM. et & Modelagem da influência do & Relatório (usando & Parto vaginal e \\
\hline
\end{tabular}




\begin{tabular}{|c|c|c|c|}
\hline al., 2017 & $\begin{array}{l}\text { contato precoce pele a pele no } \\
\text { aleitamento materno } \\
\text { exclusivo em uma amostra de } \\
\text { mulheres imigrantes } \\
\text { hispânicas }^{(25)} \text {. }\end{array}$ & $\begin{array}{l}\text { dados de um estudo } \\
\text { longitudinal) } \\
6\end{array}$ & cesariana. \\
\hline $\begin{array}{l}\text { Safari K; Saeed } \\
\text { AA; Hasan SS; } \\
\text { Banaem LM; } \\
2018\end{array}$ & $\begin{array}{l}\text { O efeito do contato pele a } \\
\text { pele precoce entre mãe e } \\
\text { recém-nascido no início da } \\
\text { amamentação, temperatura do } \\
\text { recém-nascido e duração do } \\
\text { terceiro estágio do trabalho }^{\text {de parto }}{ }^{(26)} \text {. }\end{array}$ & $\begin{array}{l}\text { Estudo quase- } \\
\text { experimental } \\
3\end{array}$ & $\begin{array}{l}\text { Início da amamentação; } \\
\text { Duração da terceira fase } \\
\text { do trabalho de parto; } \\
\text { Hipotermia neonatal. }\end{array}$ \\
\hline $\begin{array}{l}\text { Cadwell K; } \\
\text { Brimdyr K; } \\
\text { Phillips R; } 2018\end{array}$ & $\begin{array}{l}\text { Mapeamento, medição e } \\
\text { análise do processo de } \\
\text { contato pele a pele e } \\
\text { aleitamento materno na } \\
\text { primeira hora após o } \\
\text { nascimento }^{(27)} \text {. }\end{array}$ & $\begin{array}{l}\text { Estudo descritivo } \\
\text { transversal } \\
4\end{array}$ & $\begin{array}{l}\text { Início da amamentação; } \\
\text { Cuidados de rotina em } \\
\text { sala de parto. }\end{array}$ \\
\hline $\begin{array}{l}\text { Brimdyr K; } \\
\text { Cadwell K; } \\
\text { Stevens J; } \\
\text { Takahashi Y; } \\
2018\end{array}$ & $\begin{array}{l}\text { Um algoritmo de } \\
\text { implementação para melhorar } \\
\text { a prática pele a pele na } \\
\text { primeira hora após o } \\
\text { nascimento }{ }^{(28)} \text {. }\end{array}$ & $\begin{array}{l}\text { Estudo quase- } \\
\text { experimental } \\
3\end{array}$ & Amamentação. \\
\hline $\begin{array}{l}\text { Karimi FZ; } \\
\text { Sadeghi R; } \\
\text { Maleki-Saghooni } \\
\text { N; Khadivzadeh } \\
\text { T; } 2018\end{array}$ & $\begin{array}{l}\text { O efeito do contato pele a } \\
\text { pele mãe-bebê no sucesso e } \\
\text { duração da primeira } \\
\text { amamentação: uma revisão } \\
\text { sistemática e meta-análise }^{(29)} \text {. }\end{array}$ & $\begin{array}{l}\text { Revisão sistemática } \\
\text { e meta-análise } \\
1\end{array}$ & $\begin{array}{l}\text { Amamentação; } \\
\text { Redução do estresse } \\
\text { materno e infantil. }\end{array}$ \\
\hline $\begin{array}{l}\text { Alenchery AJ. et } \\
\text { al., } 2018\end{array}$ & $\begin{array}{l}\text { Barreiras e facilitadores ao } \\
\text { contato pele a pele ao } \\
\text { nascimento em recém- } \\
\text { nascidos saudáveis - um } \\
\text { estudo qualitativo }^{(30)} \text {. }\end{array}$ & $\begin{array}{l}\text { Estudo qualitativo } \\
4\end{array}$ & $\begin{array}{l}\text { Atuação da equipe de } \\
\text { sala de parto. }\end{array}$ \\
\hline $\begin{array}{l}\text { Shaw SC; Devgan } \\
\text { A; Anila S; } \\
\text { Anushree MN; } \\
\text { Debnath H; } 2018\end{array}$ & $\begin{array}{l}\text { Uso de ciclos Planejar-Fazer- } \\
\text { Estudar-Agir (PDSA) para } \\
\text { diminuir a incidência de } \\
\text { hipotermia neonatal na sala } \\
\text { de parto }^{(31)} \text {. }\end{array}$ & $\begin{array}{l}\text { Estudo quase- } \\
\text { experimental } \\
3\end{array}$ & Hipotermia neonatal. \\
\hline $\begin{array}{l}\text { Abdala LG; Da } \\
\text { Cunha MLC; } \\
2018\end{array}$ & 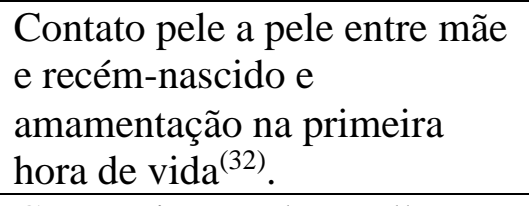 & $\begin{array}{l}\text { Estudo transversal } \\
4\end{array}$ & $\begin{array}{l}\text { Parto vaginal e } \\
\text { cesariana. }\end{array}$ \\
\hline $\begin{array}{l}\text { Thuler ACMC; } \\
\text { Wall ML; De } \\
\text { Souza MAR; } \\
2018\end{array}$ & $\begin{array}{l}\text { Caracterização das mulheres } \\
\text { no ciclo gravídico-puerperal e } \\
\text { o incentivo à amamentação } \\
\text { precoce }^{(33)} \text {. }\end{array}$ & $\begin{array}{l}\text { Estudo quantitativo, } \\
\text { descritivo, } \\
\text { longitudinal } \\
4\end{array}$ & $\begin{array}{l}\text { Parto vaginal e } \\
\text { cesariana. }\end{array}$ \\
\hline $\begin{array}{l}\text { Bedaso A; } \\
\text { Kebede E; } \\
\text { Adamu T; } 2019\end{array}$ & $\begin{array}{l}\text { Avaliação do contato pele a } \\
\text { pele (CSC) durante a } \\
\text { internação pós-parto e seus } \\
\text { fatores determinantes entre } \\
\text { mães de instituições de saúde }\end{array}$ & $\begin{array}{l}\text { Estudo transversal } \\
4\end{array}$ & $\begin{array}{l}\text { Nível educacional das } \\
\text { mulheres e número de } \\
\text { consultas de pré-natal. }\end{array}$ \\
\hline
\end{tabular}


Fonte: Elaboração própria

A análise dos estudos baseou-se no objetivo e na pergunta norteadora desta revisão. Identificou-se fatores fisiológicos que estavam relacionados à mãe e ao bebê durante a ocorrência do CPP e da mesma forma foram notados comportamentos que interferiam nesta prática de forma positiva ou negativa. Assim sendo, para melhor organização e discussão dos achados foram criadas duas categorias. São elas: Fatores comportamentais e Fatores fisiológicos.

\section{DISCUSSÃO}

\section{Fatores comportamentais}

Essa categoria originou-se a partir de estudos que apontam quais comportamentos são iniciados ou favorecidos a partir da prática do CPP na primeira hora de vida e por outro lado, quais comportamentos maternos, infantis ou assistenciais impedem que a prática seja realizada de acordo com as principais recomendações dos órgãos de saúde.

$\mathrm{O}$ ato que colocar o recém-nascido no peito desnudo da mãe assim que ele nasce favorece a primeira mamada e isso permite que a mulher veja, toque e pegue no seu filho, suprindo assim, toda a expectativa criada durante as semanas da gestação, o que fortalece o vínculo afetivo entre os dois ${ }^{(18)}$. Observou-se que puérperas que não vivenciaram o CPP com seus filhos na primeira hora de vida eram significativamente https://doi.org/10.31011/reaid-2021-v.95-n.35-art.1079 Rev Enferm Atual In Derme v. 95, n. 35, 2021 e-021120 mais tristes dos que aquelas que tiveram a oportunidade. Isso mostra que a falta do CPP influencia negativamente no vínculo entre mãe e filho ${ }^{(24)}$. A separação precoce de mãe e bebê pode diminuir a resposta afetiva da mãe ao seu filho e isso pode gerar efeito negativo no comportamento materno ${ }^{(17)}$.

As práticas profissionais foram apontadas como fatores dificultadores para a realização do CPP na sala de parto. Observou-se que boa parte dos binômios mãe-filho realizaram contato pele-pano ou contato misto durante a primeira hora de vida, sendo que a mediana de duração do CPP foi de 30 minutos apenas. Este mesmo estudo defende que deve acontecer a interação entre mãe e bebê na primeira hora de vida e que para isso o papel da equipe multiprofissional que atende ao parto e nascimento é bastante relevante ${ }^{(32)}$.

A influência da equipe de saúde no CPP também é evidenciada em outros estudos que apontam que a separação do binômio na primeira hora pós parto ocorre em prol da realização de cuidados de rotina com mães e principalmente com o recém-nascido ${ }^{(16)}$. Isso ocorre quando os profissionais de saúde não percebem relevância em promover $\mathrm{CPP} e$ aleitamento materno na primeira hora pósparto. Dessa forma permitem o primeiro contato entre mãe e bebê de maneira rápida e mecânica para que o fluxo de suas atividades na sala de parto não seja prejudicado ou 
atrasado. Autores apontam que prevenir a separação da díade mãe-filho em ocasiões em que ambos se encontram saudáveis é uma responsabilidade ética dos profissionais de saúde ${ }^{(17)}$.

Ainda no que se refere aos cuidados prestados em sala de parto, ressalta-se que o Ministério da Saúde do Brasil recomenda que seja adiado qualquer cuidado de rotina que separe mãe e filho desde que o binômio se encontre estável. $\mathrm{O}$ objetivo deste adiamento é justamente propiciar contato ininterrupto na primeira hora após o nascimento ${ }^{(2)}$.

Estudo conduzido em Bangalore, Índia, no ano de 2018, elencou barreiras e facilitadores para o CPP no nascimento. Como fatores que motivaram a promoção do CPP tiveram: conhecimento dos benefícios da prática, experiências positivas vivenciadas pelos profissionais anteriormente àquele momento, e por fim, aceitação materna. As principais barreiras notadas no estudo foram: falta de pessoal na equipe de enfermagem, restrição de tempo alegada pelos profissionais da sala de parto que acreditavam que a prática do $\mathrm{CPP}$ provocaria atraso às outras atividades do setor e dessa forma consideravam o tempo de 1 hora impraticável, apreensões relacionadas ao procedimento como preocupação com a segurança do RN no colo da mãe, intolerância com rotinas clínicas e dificuldades em decidir sobre a elegibilidade para $\mathrm{CPP}^{(30)}$.

Quando falou sobre cuidados essenciais ao recém-nascido no ano de 2003, Zveiter defendeu que a primeira hora após o nascimento da criança é uma hora delicada onde a mãe encontra-se ainda impactada pela chegada do seu bebê. Nesse contexto, a equipe de saúde envolvida no nascimento representa uma figura facilitadora ou não do processo de aproximação precoce entre a mãe e seu filho. Visto isso, as ações desses profissionais de saúde no pós-parto imediato em relação aos cuidados prestados ao recémnascido podem interferir negativamente no vínculo que está se estabelecendo entre os dois seres ${ }^{(35)}$.

Portanto, os profissionais que atuam na recepção do recém-nascido devem entender que, na verdade, são coadjuvantes do processo do nascimento e as práticas chamadas de rotineiras da assistência só devem ser trocadas por técnicas de atendimentos imediatos de urgência, pois, caso contrário, se tornarão intervenções desnecessárias e prejudiciais à primeira hora de vida do recém-nascido ${ }^{(36)}$.

Uma equipe qualificada para o atendimento à mulher na sala de parto consegue garantir a realização do $\mathrm{CPP}$, pois uma equipe treinada e preparada contribui para que a indicação de intervenções ao parto e as avaliações da condição de nascimento da

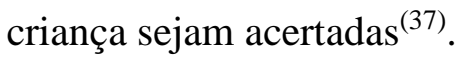

Seis estudos selecionados associaram a prática do CPP com o início e a duração da amamentação. O CPP imediato e ininterrupto apresenta efeito positivo e significativo na 
taxa de sucesso e na duração da primeira lactação ${ }^{(26,27,29)}$. Além disso, está associado a um aumento nas chances de aleitamento materno exclusivo no momento da alta ${ }^{(25)}$. De encontro a esses achados, um estudo aponta que o CPP melhora significativamente a taxa de aleitamento materno exclusivo às seis semanas de idade em neonatos saudáveis a termo $^{(21)}$. Interromper ou não realizar o CPP após o nascimento pode inibir o reflexo de rastreamento pelo recém-nascido, fazendo com o que o início da amamentação seja postergado $^{(17)}$.

A cesariana foi apontada como fator dificultador para a realização do CPP e associada a realização de contato pele-pano na primeira hora de vida. A maioria das puérperas submetidas à cesariana não teve o contato pele-a-pele na sala de parto, sendo inverso nos casos de parto normal. Isso reflete no aleitamento materno, uma vez que bebês nascidos de cesariana têm uma taxa menor de amamentação na alta hospitalar em comparação com aqueles nascidos de parto vaginal. $\mathrm{O}$ tipo de parto é o fator que mais influencia na realização do $\operatorname{CPP}(23,25,32,33)$.

O CPP se faz muito importante na cesariana para proporcionar, também nesta via de parto, um momento íntimo, de troca afetiva, estímulos e calor. O CPP deve ser estimulado na cesariana para o conforto e desenvolvimento saudável do recém-nascido. É muito valioso que a equipe assistente apoie este momento em que a mulher passa a ser mãe e nutriz. São esses profissionais que proporcionam o início do contato e auxiliam o binômio neste primeiro momento juntos agora fora do ventre ${ }^{(38-40)}$.

\section{Fatores fisiológicos}

Esta categoria emergiu-se em estudos da amostra que mencionam reações/efeitos/respostas dos organismos materno e infantil que são decorrentes da realização do CPP.

Manter mãe e bebê juntos após o parto contribui para a manutenção da temperatura corporal do recém-nascido ${ }^{(20)}$. E ainda sobre temperatura corporal do recém-nascido, outros três estudos desta revisão comprovam que a realização do CPP diminui as taxas de incidência de hipotermia neonatal. Além de ser uma alternativa adequada e de alta qualidade, o CPP é também facilmente implementado em qualquer estabelecimento de saúde, independente do porte ${ }^{(17,26,31)}$.

Outros benefícios foram observados e comprovados com a prática do CPP entre mãe e filho após o nascimento. Um exemplo são mães que vivenciaram o CPP imediatamente após o parto e apresentaram escore de dor durante reparo de lacerações consideravelmente menor do que aquelas que não viveram o contato ${ }^{(21)}$. Outro benefício demonstrado nessa categoria foi o prazer que os hormônios liberados no parto proporcionam à mãe que recebe o seu filho no colo imediatamente ${ }^{(17)}$. 
Durante o CPP o organismo materno libera ocitocina que por sua vez é um hormônio que colabora com a contratilidade uterina no pós-parto imediato prevenindo, assim, hemorragias por atonia uterina ${ }^{(9)}$. A duração da terceira fase do trabalho de parto em mães que praticaram CPP após o nascimento foi menor em comparação com mães que receberam cuidados de rotina ${ }^{(26)}$.

Ainda no que se refere ao risco de hemorragia na primeira hora pós-parto, um estudo australiano teve como resultado que, devido a liberação endógena de ocitocina, as mulheres que viveram o CPP e amamentaram no pós-parto imediato, tiveram duas vezes menos chances de sofrer hemorragia pósparto primária (HPP) em comparação com mulheres que não vivenciaram o CPP e não amamentaram na primeira hora de vida do bebê. E por fim, os autores sugeriram que o CPP e a amamentação imediatamente após o nascimento são medidas que podem ser importantes e de alta eficácia na redução das taxas de HPP para mulheres ${ }^{(11)}$.

É comprovado que o CPP acalma o binômio mãe-filho que entram em sintonia naquele primeiro momento de vida extrauterina da criança. Além disso, regula a respiração e a frequência cardíaca de ambos, coloniza o bebê com a flora bacteriana materna e reduz o choro, reduzindo assim o estresse do bebê. As bactérias advindas do corpo da mãe protegem os bebês de infecções e ajudam a construir seu sistema imunológico.
É, portanto, importante que o CPP seja iniciado imediatamente após o nascimento e seja contínuo, prolongado e estabelecido entre toda a mãe-filho saudáveis ${ }^{(9)}$.

\section{CONCLUSÃO}

Os fatores associados ao CPP encontrados a partir dessa revisão foram: fatores comportamentais (atuação da equipe de sala de parto, vínculo materno infantil, início e duração da amamentação, tipo de parto) e fisiológicos (bem-estar materno e infantil no pós-parto imediato, termorregulação neonatal, redução de estresse e dor do binômio e prevenção de hemorragia no pós-parto).

Através deste estudo foi possível observar a diversidade de benefícios que a prática do CPP promove para o binômio mãe-bebê. Observou-se também quais são os fatores que dificultam ou impedem a realização desse contato.

O CPP vem ganhando espaço nas salas de parto ao redor do mundo, mas ainda existem falhas no modo de fazê-lo e há resistência por parte de alguns profissionais que indagam sobre a sua eficácia e sobre a real necessidade uma vez que acreditam que a prática pode atrasar a dinâmica do setor.

É inquestionável a importância de todos os profissionais que atuam $24 \mathrm{~h}$ por dia no cenário de parto e nascimento. Todos são peças importantes e facilitadoras do CPP na primeira hora de vida e, portanto, é essencial que façam parte de uma equipe treinada, 
capacitada e conhecedora da aplicabilidade do primeiro contato entre mãe e filho imediatamente após o nascimento.

\section{REFERÊNCIAS}

1. Cruz DCS, Sumam NDS, Spíndola T. Os cuidados imediatos prestados ao recémnascido e a promoção do vínculo mãe-bebê. Rev. esc. enferm. USP [Internet]. 2007 [acesso em 26 fev 2020]; 41(4): 690-97. Disponível

em: http://www.scielo.br/scielo.php?script=sci_art text\&pid=S008062342007000400021\&lng=e

2. Ministério da Saúde (Brasil), Secretaria de Atenção à Saúde. Departamento de Ações Programáticas e Estratégicas. Além da sobrevivência: práticas integradas de atenção ao parto, benéficas para a nutrição e a saúde de mães e crianças / Ministério da Saúde, Secretaria de Atenção à Saúde, Área Técnica de Saúde da Criança e Aleitamento Materno. - 1. ed., 1. reimp. - Brasília : Ministério da Saúde, 2013.

3. Moore ER, Bergman N, Anderson GC, Medley N. Early skin-to-skin contact for mothers and their healthy newborn infants. Cochrane Database of Systematic Reviews 2016, Issue 11. Art. No.:

4. Ministério da saúde (Brasil), Governo do Brasil - Saúde atualiza diretrizes para atenção humanizada a recém-nascido. 2017. Disponível em: http://www.brasil.gov.br/noticias/saude/2014/ 05/saude-atualiza-diretrizes-paraatencaohumanizada-a-recem-nascido Acessado 28/02/2020

5. Monte GCSB, Leal LP, Pontes CM. Avaliação do $4^{\circ}$ passo para promoção do aleitamento materno em hospital amigo da criança. Rev Rene [Internet]. 2012; [acesso em 2020 Fev 26]; 13(4): 861-870. Disponível em:

https://www.redalyc.org/articulo.oa?id=32402 $\underline{7983016}$
6. World Health Organization. Guideline: protecting, promoting and supporting breastfeeding in facilities providing maternity and newborn services. WHO [Internet] 2017 [cited 2020 Feb 26]. Available from: http://www.who.int/nutrition/publications/gui delines/breastfeeding-facilities-maternitynewborn/en/

7. Ministério da Saúde (Brasil), Área Técnica de Saúde da Criança e Aleitamento Materno, Departamento de Ações Programáticas Estratégicas, Secretaria de Atenção à Saúde. Iniciativa Hospital Amigo da Criança. Brasília: Ministério da Saúde; 2011

8. Selim L. Breastfeeding from the first hour of birth: What works and what hurts. [Internet]; 201831 July. [acesso em 2020 Fev 28]. Disponível em: https://www.unicef.org/stories/breastfeedingfirst-hour-birth-what-works-and-what-hurts

9. Ministério da Saúde (Brasil), Fundo das Nações Unidas para a Infância/Organização Mundial da Saúde. Iniciativa hospital amigo da criança: Revista, atualizada e ampliada para o cuidado integrado. Série A. Normas e Manuais Técnicos. Brasília (DF): Ministério da Saúde; 2009

10. Shidu S, Garwood P. 3 in 5 babies not breastfed in the first hour of life. Breastfeeding within an hour after birth is critical for saving newborn lives [Press release]. 201830 July. [Acesso em 2020 Fev 28] Disponível em: https://www.unicef.org/press-releases/3-5babies-not-breastfed-first-hour-life

11. Saxton A, Fahy K, Rolfe M, Skinner V, Hastie C. Midwifery. Does skin-to-skin contact and breast feeding at birth affect the rate of primary postpartum haemorrhage: Results of a cohort study. Epub [Internet]. 2015; [cited 2020 Feb 28]; 31(11): 1110-7 Available from: https://www.ncbi.nlm.nih.gov/pubmed/26277 $\underline{824}$

12. Narchi NZ, Fernandes Rosa Áurea Quintella, Dias Lílian de Araújo, Novais Daniela Higasa. Variáveis que influenciam a manutenção do aleitamento materno exclusivo. Rev. esc. enferm. USP [Internet]. 
2009 Mar [cited 2020 Feb 26] ; 43( 1 ): 8794. Available from: http://www.scielo.br/scielo.php?script=sci_art text\&pid=S0080-

62342009000100011\&lng=en.

13. Matos TA, Souza MS, Santos EKA, Velho MB, Seibert ERC, Martins NM. Contato precoce pele a pele entre mãe e filho: significado para mães e contribuições para a enfermagem. Rev. bras. enferm. [Internet]. 2010 Dec [acesso em 26 fev 2020] ; 63(6): 998-1004. Disponível em: http://www.scielo.br/scielo.php?script=sci_art text\&pid=S0034-

$71672010000600020 \& \operatorname{lng}=\mathrm{en}$.

14. Souza MT, Silva MD, Carvalho R. Revisão integrativa: o que é e como fazer. Einstein. 2010; 8(1): 102-6.

15. Galvão CM, Sawada NO, Mendes Isabel Amélia Costa. A busca das melhores evidências. Rev. esc. enferm. USP [Internet]. 2003 [acesso em 26 fev 2020]; 37 (4): 43-50. Disponível em: http://www.scielo.br/scielo.php?script=sci_art text\&pid=S0080$62342003000400005 \& \operatorname{lng}=\mathrm{en}$.

16. Dos Santos LM, Da Silva JCR, Carvalho ESS, Carneiro AJS, De Santana RCB, Fonseca MCC. Vivenciando o contato pele a pele com o recém-nascido no pós-parto como um ato mecânico. Rev. Brasileira de Enferm [Internet]. 2014 [acesso em 05 set 2019]; 67 (2): 202-7. Disponível em: https://www.scielo.br/pdf/reben/v67n2/00347167-reben-67-02-0202.pdf

17. Crenshaw JT. Healthy birth practice\# 6: Keep mother and baby together - It's best for mother, baby, and breastfeeding. The Journal of perinatal education [Internet] 2014 [acesso em 20 nov 2019]; 23 (4): 211-17. Disponível em:

https://connect.springerpub.com/content/sgrjp e/23/4/211.abstract

18. Fucks IS, Soares MC, Kerber NPC, Meincke SMK, Escobal APL, Bordignon SS. A sala de parto: o contato pele a pele e as ações para o estímulo ao vínculo entre mãe-bebê. Avances en Enfermería [Internet] 2015 [acesso em 29 out 2019]; 33(1): 29-37. Disponível em: https://revistas.unal.edu.co/index.php/avenfer $\mathrm{m} /$ article/view/47371

19. Vieira MJO, Dos Santos AAP, Silva JMO, Sanches METL. Assistência de enfermagem obstétrica baseada em boas práticas: do acolhimento ao parto. Ver Eletrônica Enferm [Internet]. 2016 [acesso em 29 out 2019]; v.18. Disponível em: https://www.revistas.ufg.br/fen/article/view/3 $\underline{6714}$

20. Albuquerque RS, Neto CM, Bersusa AAS, Dias VM, Silva MIM. Newborns' temperature submitted to radiant heat and to the Top Maternal device at birth. Rev. Latino-Am. Enfermagem [Internet]. 2016 [acesso em 29 out 2019]; 24:e2741. Disponível em: https://www.scielo.br/scielo.php?pid=S0104 $11692016000100368 \&$ script $=$ sci_arttext

21. Sharma A. Efficacy of early skin-to-skin contact on the rate of exclusive breastfeeding in term neonates: A randomized controlled trial. African Health Sciences [Internet]. 2016 [acesso em 29 out 2019]; 16(3): 790-97. Disponível em https://www.ajol.info/index.php/ahs/article/vi ew/146092

22. Silva CM, Pereira SCL, Passos IR, Dos Santos LC. Fatores associados ao contato pele a pele entre mãe/filho e amamentação na sala de parto. Rev. Nutr. [Internet]. 2016 [acesso em 16 jul 2019]; 29(4): 457-71. Disponível em:

https://www.scielo.br/scielo.php?pid=S14155 $2732016000400457 \&$ script $=$ sci_arttext

23. Guala A, Boscardini L, Visentin R, Angellotti $\mathrm{P}$, Grugni L, Barbaglia M, et. al. Skin-to-Skin Contact in Cesarean Birth and Duration of Breastfeeding: A Cohort Study. The Scientific World Journal [Internet]. 2017 [acesso em 24 out 2019]; v. 2017. 5 pages. Disponível em: https://www.hindawi.com/journals/tswj/2017/ 1940756/\#copyright

24. Souza LH, Soler ZASG, Santos MLSG, Sasaki NSGMD. Puerperae bonding with their children and labor experiences. Investigacion $\mathrm{y}$ educacion en enfermeria [Internet]. 2017 [acesso em 28 out 2019]; 35(3): 364-70. Disponível em: 
https://revistas.udea.edu.co/index.php/iee/arti cle/view/329215

25. Linares AM, Wambach K, Rayens, MK, Wiggins A, Coleman E, Dignan MB. Modeling the influence of early skin-to-skin contact on exclusive breastfeeding in a sample of Hispanic immigrant women. Journal of immigrant and minority health. [Internet]. 2017 [acesso em 28 out 2019]; 19(5): 1027$34 . \quad$ Disponível em: https://link.springer.com/article/10.1007/s109 03-016-0380-8

26. Safari K, Saeed AA, Hasan AA, MoghaddamBanaem L. The effect of mother and newborn early skin-to-skin contact on initiation of breastfeeding, newborn temperature and duration of third stage of labor. International Breastfeeding Journal [Internet]. 2018 [acesso em 24 out 2019]; 13(32): 1-8. Disponível em: https://internationalbreastfeedingjournal.biom edcentral.com/articles/10.1186/s13006-0180174-9\#citeas

27. Cadwell K, Brimdyr K, Phillips R. Mapping, measuring, and analyzing the process of skinto-skin contact and early breastfeeding in the first hour after birth. Breastfeeding Medicine [Internet]. 2018 [acesso em 28 out 2019]; 13(7): 485-92. Disponível em: https://www.liebertpub.com/doi/full/10.1089/ bfm.2018.0048

28. Brimdyr K, Cadwell K, Stevens J, Takahashi Y. An implementation algorithm to improve skin-to-skin practice in the first hour after birth. Maternal \& child nutrition [Internet]. 2018 [acesso em 28 out 2019]; 14(2): e12571. Disponível em: https://onlinelibrary.wiley.com/doi/full/10.11 $\underline{11 / \mathrm{mcn} .12571}$

29. Karimi FZ, Sadeghi R, Maleki-Saghooni N, Khadivzadeh T. The effect of mother-infant skin to skin contact on success and duration of first breastfeeding: A systematic review and meta-analysis. Taiwanese Journal of Obstetrics and Gynecology [Internet]. 2019 [acesso em 28 out 2019]; 58(1): 1-9. Disponível em: https://www.sciencedirect.com/science/article /pii/S1028455918302766
30. Alenchery AJ, Thoppil J, Britto CD, de Onis JV, Fernandez L, Rao PS. Barriers and enablers to skin-to-skin contact at birth in healthy neonates-a qualitative study. BMC pediatrics [Internet]. 2018 [acesso em 28 out 2019]; 18(1): 1-10. Disponível em: https://link.springer.com/article/10.1186/s128 87-018-1033-y

31. Shaw SC, Devgan A, Anila S, Anushree N, Debnath H. Use of Plan-Do-Study-Act cycles to decrease incidence of neonatal hypothermia in the labor room. Medical Journal Armed Forces India [Internet]. 2018 [acesso em 08 nov 2019]; 74(2): 126-32. Disponível em: https://www.sciencedirect.com/science/article /abs/pii/S0377123717300692

32. Abdala LG, Cunha MLCD. Contato pele a pele entre mãe e recém-nascido e amamentação na primeira hora de vida. Clinical and biomedical research. [Internet]. 2018 [acesso em 28 out 2019]; 38(4): 356-60. Disponível em: https://www.lume.ufrgs.br/bitstream/handle/1 0183/210480/001091458.pdf?sequence $=1$

33. Thuler ACDMC, Wall ML, de Souza MAR. Caracterização das mulheres no ciclo gravídico-puerperal e o incentivo à amamentação precoce. Revista Enfermagem UERJ [Internet]. 2018 [acesso em 29 out 2019]; 26:16936. Disponível em: https://www.e-

publicacoes.uerj.br/index.php/enfermagemuer $\mathrm{j} /$ article/view/16936

34. Bedaso A, Kebede E, Adamu T. Assessment of skin-to-skin contact (SSC) during the postpartum stay and its determinant factors among mothers at public health institutions in Ethiopia. BMC research notes [Internet]. 2019 [acesso em 28 out 2019]; 12(1): 1-7. Disponível em: https://bmcresnotes.biomedcentral.com/article s/10.1186/s13104-019-4176-5

35. Zveiter, M. Contribuições ao documento da Organização Mundial de Saúde (1986): cuidados essenciais ao recém-nascido comentário sobre as implicações psíquicas. [Dissertação]. Rio de Janeiro: Instituto Fernandes Figueira, Fundação Oswaldo Cruz; 2003. 
36. de Oliveira LSH, Goulart MJP, Carmo TMD. Assistência ao recém-nascido pelos profissionais de enfermagem na sala de parto no momento da recepção. Ciência Et. Praxis [Internet]. 2010 [acesso em $26 \mathrm{fev}$ 2020]; 3(5): 35-42. Disponível em: http://revista.uemg.br/index.php/praxys/article /view/2158/1150

37. Saco MC, Coca KP, Marcacine KO, Abuchaim EDSV, Abrão ACFV. Contato pele a pele e mamada precoce: fatores associados e influência no aleitamento materno exclusivo. Texto contexto - enferm. [Internet]. 2019 [acesso em 28 fev 2020]; 28: e20180260. Disponível em: http://www.scielo.br/scielo.php?script=sci_art text\&pid=S0104-

07072019000100391\&lng=en.

O desenvolvimento deste artigo contou com a colaboração de algumas pessoas, dentre as quais agradecemos:

As acadêmicas de Enfermagem Caroline Souza Amaral e Thalita Botelho Cutrim

Submissão: 2021-04-12

Aprovado: 2021-05-02
38. Marques Anelise Niederauer. O que diz a literatura sobre contato pele a pele entre a mãe e o recém-nascido durante a cesariana: em busca de argumentos para as boas práticas na atenção ao nascimento. [trabalho de conclusão de curso de especialização]. Porto Alegre: Universidade Federal Do Rio Grande Do Sul; 2016

39. Monteiro JCS. Contato precoce e amamentação em sala de parto na perspectiva da mulher [Dissertação]. Ribeirão Preto: Escola de Enfermagem de Ribeirão Preto, Universidade de São Paulo; 2006.

40. Merighi MAB, Gonçalves R, Rodrigues IG. Vivenciando o período puerperal: uma abordagem compreensiva da Fenomenologia Social. Rev Bras Enferm. 2006; 59(6): 775-9. 